\title{
GCU
}

Glasgow Caledonian

University

University for the Common Good

\section{The contribution of alcohol use disorder to decompensated cirrhosis among people with hepatitis $\mathbf{C}$}

Alavi, Maryam ; Janjua, Naveed Z. ; Chong, Mei; Grebely, Jason; Aspinall, Esther J.; Innes, Hamish; Valerio, Heather M.; Hajarizadeh, Behzad ; Hayes, Peter C.; Krajden, Mel; Amin, Janaki; Law, Matthew G.; George, Jacob; Goldberg, David J.; Hutchinson, Sharon J.; Dore, Gregory J.

Published in:

Journal of Hepatology

DOI:

10.1016/j.jhep.2017.10.019

Publication date:

2018

Document Version

Author accepted manuscript

Link to publication in ResearchOnline

Citation for published version (Harvard):

Alavi, M, Janjua, NZ, Chong, M, Grebely, J, Aspinall, EJ, Innes, H, Valerio, HM, Hajarizadeh, B, Hayes, PC, Krajden, M, Amin, J, Law, MG, George, J, Goldberg, DJ, Hutchinson, SJ \& Dore, GJ 2018, 'The contribution of alcohol use disorder to decompensated cirrhosis among people with hepatitis C', Journal of Hepatology, vol. 68, no. 3, pp. 393-401. https://doi.org/10.1016/j.jhep.2017.10.019

\section{General rights}

Copyright and moral rights for the publications made accessible in the public portal are retained by the authors and/or other copyright owners and it is a condition of accessing publications that users recognise and abide by the legal requirements associated with these rights.

Take down policy

If you believe that this document breaches copyright please view our takedown policy at https://edshare.gcu.ac.uk/id/eprint/5179 for details of how to contact us. 
The contribution of alcohol-use disorder to decompensated cirrhosis among people with hepatitis $\mathrm{C}$ : an international comparison

Maryam Alavi ${ }^{1,2,3}$, Naveed Z. Janjua ${ }^{4,5}$, Mei Chong ${ }^{4}$, Jason Grebely ${ }^{1}$, Esther J. Aspinall ${ }^{2,3}$, Hamish Innes ${ }^{2,3}$, Heather M. Valerio ${ }^{2,3}$, Behzad Hajarizadeh ${ }^{1}$, Peter C. Hayes ${ }^{6}$, Mel Krajden $^{4,7}$, Janaki Amin ${ }^{1,8}$, Matthew G. Law ${ }^{1}$, Jacob George ${ }^{9}$, David J. Goldberg ${ }^{2,3}$, Sharon J. Hutchinson $^{2,3}$, Gregory J. Dore ${ }^{1}$

${ }^{1}$ The Kirby Institute, UNSW Sydney, Sydney, NSW, Australia; ${ }^{2}$ School of Health and Life Sciences, Glasgow Caledonian University, Glasgow, UK; ${ }^{3}$ Health Protection Scotland, National Services Scotland, Glasgow, UK; ${ }^{4}$ British Columbia Centre for Disease Control, Vancouver, British Columbia, Canada; ${ }^{5}$ School of Population and Public Health, University of British Columbia, Vancouver, British Columbia, Canada; ${ }^{6}$ Royal Infirmary Edinburgh, Edinburgh, UK; ${ }^{7}$ Department of Pathology and Laboratory Medicine, University of British Columbia, Vancouver, BC, Canada; ${ }^{8}$ Department of Health Systems and Populations, Macquarie University, Sydney, NSW, Australia; ${ }^{9}$ Storr Liver Centre, Westmead Millennium Institute for Medical Research, University of Sydney and Westmead Hospital, Westmead, Australia 


\section{Corresponding Author}

Maryam Alavi

Viral Hepatitis Clinical Research Program

The Kirby Institute

UNSW Sydney

Mailing address:

The Kirby Institute

Wallace Wurth Building

UNSW Sydney

NSW Australia 2052

Telephone: +61 (2) 93850881 - Facsimile: +61 (2) 93850876.

Email address: msalehialavi@kirby.unsw.edu.au 
Word Count (including the abstract, references, tables and figure legends)

4,886

Tables

4

Figures

2

List of abbreviations in the order of appearance

hepatitis $\mathrm{C}$ virus (HCV); direct acting antiviral (DAA); World Health Organization (WHO); alcohol-use disorder (AUD); British Columbia (BC); New South Wales (NSW); decompensated cirrhosis (DC); British Columbia Hepatitis Testers Cohort (BC-HTC); Classification of Diseases and Related Health Problems (ICD-10); Notifiable Conditions Information Management System (NCIMS); person-years (PY); population attributable fraction (PAF); adjusted hazard ratio (aHR).

Keywords

$\mathrm{HCV}$; liver disease; alcohol-use disorder; population-based; data linkage 


\section{Conflict of interest statements}

J Grebely has received research support and personal fees from Gilead Sciences and Merck. J Grebely has received research support from Abbvie, Bristol-Myers Squibb, and Cepheid. HI has received personal fees from Gilead Sciences. PCH has received research support and personal fees from Roche. PCH has received personal fees from MSD, Gilead Sciences, Abbvie, Janssen, Bristol-Myers Squibb, Pfizer, and Novartis. MK has received research support (via his institution) from Roche, Boehringer Ingelheim, Merck, Siemens Healthcare Diagnostics, and Hologic Inc. MGL has received research support from Boehringer Ingelheim, Merck, Bristol-Myers Squibb, Janssen, and ViiV HealthCare. MGL has received research support and personal fees from Gilead Sciences. MGL has received personal fees from Sirtex Pty Ltd. J George has received personal fees from MSD, Gilead Sciences, Bristol-Myers Squibb, Abbvie, and Pharmaxis. DJG has received personal fees from Gilead Sciences, Merck, and Abbvie. GJD has received research and travel support, and personal fees from Abbvie, Merck, Bristol-Myers Squibb, and Roche. GJD has received research support and personal fees from Janssen. GJD has received personal fees and travel support from Gilead Sciences. GJD has received personal fees from GlaxoSmithKline and Abbott Diagnostics. Other authors have no commercial relationships that might pose a conflict of interest in connection with this manuscript. 


\section{Financial support statement}

The BC component of this work was supported by British Columbia Centre for Disease Control and the Canadian Institutes of Health Research [Grant \# 201410PHE-337680-PHECAAA-179547]. The authors acknowledge the assistance of BC Centre for Disease Control, Provincial Health Services Authority Performance measurement and reporting, Information Analysts, BC Ministry of Health Data Access, Research and Stewardship, MSP, Discharge Abstract Database, and Medical Beneficiary and Pharmaceutical Services programme areas, BC Ministry of Health, and BC Cancer Agency and their staff involved in data access and procurement, and data management. The NSW component of this work was supported by the Kirby Institute. Kirby Institute is funded by the Australian Government Department of Health, under the agreement ID number 2-D3X513. This publication is part of the Bloodborne viruses and sexually transmissible infections Research, Strategic Interventions and Evaluation (BRISE) program, funded by the NSW Ministry of Health. This publication has received funding from the Sydney West Translational Cancer Research Centre (SWTCRC) program "HOTTer-West: HCC Outcome improvements Through Translational research in WESTern Sydney. The Scottish component of this work was funded by the Scottish Government. The authors acknowledge the assistance of Health Protection Scotland, Information Services Division, Hepatitis C Clinical Database Monitoring Committee, the clinics and staff supporting the Hepatitis C Clinical Database, and the laboratories and staff supporting the Hepatitis $\mathrm{C}$ diagnosis and testing databases.

MA is the recipient of a CASCADE International Fellowship. The research leading to these results has received funding from the People Programme (Marie Curie Actions) of the European Union's Seventh Framework Programme (FP7/2007-2013) under REA grant agreement No PCOFUND-GA-2012-600181. J Grebely is supported by a National Health 
and Medical Research Council (NHMRC) of Australia Career Development Fellowship. BH is supported by an NHMRC of Australia Early Career Fellowship. GJD is supported through an NHMRC of Australia Practitioner Fellowships. J George is supported by an NHMRC of Australia Program Grant (1053206), project grants (632630, 1049857), a Sydney West Translational Cancer Research Centre grant funded by the Cancer Institute NSW, and the Robert W. Storr Bequest to the Sydney Medical Foundation, University of Sydney

\section{Ethics statement}

This publication involved information already collected by population-based health administration registries; therefore, people have not been 'recruited' for the purposes of this research. A waiver of consent has been approved for each component of this work, by the relevant ethics committees in $\mathrm{BC}$ and NSW, and the Scottish Government. The BC component of this work has received ethics approval from the Behavioural Research Ethics Board, Office of Research Services, University of British Columbia (reference number H1401649). The NSW component of this work has received ethics approval from NSW Population \& Health Services Research Ethics Committee, Cancer Institute NSW (reference number HREC/13/CIPHS/63). The Scottish component of this work has utilised information

within the surveillance framework of National Services Scotland. Approval for updating the linkage was granted by the National Services Scotland Public Benefit Privacy Panel.

\section{Author contributions}

MA, NZJ, and GJD contributed to study conception and design, data acquisition and analysis, interpretation of findings, and drafting of the manuscript; MC, J Grebely, EJA, HI, HMV, $\mathrm{BH}$, and JA contributed to data acquisition and analysis and interpretation of findings, and; 
PCH, MK, MGL, J George, DJG, and SJH contributed to study conception and design, data acquisition and analysis, and interpretation of findings.

\section{Disclaimer}

All inferences, opinions, and conclusions drawn in this publication are those of the author(s), and do not necessarily reflect the opinions or policies of the British Columbia Ministry of Health, Australian Government Department of Health, or the Scottish Government. 


\section{Abstract (261 words)}

Background: The aim of this study was to evaluate the contribution of alcohol-use disorder to hepatitis $\mathrm{C}$ virus (HCV)-related decompensated cirrhosis diagnosis in three settings. Methods: HCV notifications from British Columbia (BC), Canada, New South Wales (NSW), Australia, and Scotland (1995-2011/2012/2013, respectively) were linked to hospital admissions (2001-2012/2013/2014, respectively). Alcohol-use disorder was defined by nonliver-related hospitalisation due to alcohol use. Age-standardised decompensated cirrhosis incidence rates were plotted, associated factors were assessed using Cox regression, and alcohol-use disorder-associated population attributable fractions (PAFs) were computed. Findings: Among 58,487, 84,529, and 31,924 people with HCV in BC, NSW, and Scotland, 2,689 (4.6\%), 3,169 (3.7\%), and 1,375 (4.3\%) had a decompensated cirrhosis diagnosis, and $28 \%, 32 \%$, and $50 \%$ of those with decompensated cirrhosis had alcohol-use disorder, respectively. Age-standardised decompensated cirrhosis incidence rates were considerably higher among people with alcohol-use disorder in NSW and Scotland. Decompensated cirrhosis was independently associated with alcohol-use disorder in BC, aHR 1.92, 95\% CI 1.76, 2.10; NSW, aHR 3.68, 95\% CI 3.38, 4.00, and; Scotland, aHR 3.88, 95\% CI 3.42, 4.40. The PAFs of decompensated cirrhosis-related to alcohol-use disorder were $13 \%, 25 \%$, and $40 \%$ in $\mathrm{BC}$, NSW, and Scotland, respectively. Interpretation: Alcohol-use disorder was a major contributor to HCV liver disease burden in all settings, more distinctly in Scotland. The extent to which alcohol use would compromise the individual and population level benefits of direct-acting antiviral therapy (DAA) needs to be closely monitored. Countries, where appropriate, must develop strategies combining DAA treatment uptake promotion and alcohol-use disorder management, if World Health Organization 2030 HCV mortality reduction targets are to be achieved. 


\section{Lay Summary}

The burden of advanced liver disease has been rising among people with hepatitis $\mathrm{C}$ globally. The prospect of increased access to direct-acting antiviral (DAA) therapies instigated the World Health Organization (WHO) to set ambitious targets for elimination of hepatitis $\mathrm{C}$ as a major public health threat by 2030 . However, continued heavy alcohol intake is likely to impact potential benefits of DAA-based cure on individual-level liver disease progression and population-level liver disease burden. The aim of this study was to evaluate the contribution of alcohol-use disorder to hepatitis C-related decompensated cirrhosis diagnosis in British Columbia, Canada, New South Wales, Australia, and Scotland. Alcohol-use disorder was a major contributor to hepatitis C-related liver disease burden in all settings, more distinctly in Scotland. The extent to which alcohol use would compromise the individual and population level benefits of DAA-based therapies needs to be closely monitored. Countries, where appropriate, must develop strategies combining DAA treatment uptake promotion and alcohol-use disorder management, if WHO 2030 hepatitis C mortality reduction targets are to be achieved. 


\section{Introduction}

The burden of advanced liver disease has been rising among people with hepatitis $\mathrm{C}$ virus (HCV) infection globally [1]. The prospect of increased access to direct-acting antiviral (DAA) therapies instigated the World Health Organization (WHO) to set ambitious targets for elimination of hepatitis $\mathrm{C}$ as a major public health threat by 2030 [2]. However, in settings with higher levels of alcohol-use disorder (AUD), the potential for HCV treatment programs to achieve the WHO liver mortality reduction target of $65 \%$ could be compromised, without developing concurrent strategies to reduce the consumption and impact of alcohol.

Action against AUD is particularly important given that people with $\mathrm{HCV}$ infection are more likely to engage in harmful consumption [3], and alcohol is a risk factor for higher liverrelated mortality [4]. However, given the limited number of population-based studies, the relative contribution of AUD to the burden of liver disease in different HCV populations is not clear. The WHO HCV elimination strategy has highlighted the need to address alcohol use as a common co-morbidity among people with HCV infection [2]; yet, reducing the impact of alcohol is not included among target areas. Restriction of access to DAA treatment on the basis of ongoing alcohol use is present in many settings [5,6], further potentially impacting treatment programs in WHO regions with higher per capita alcohol consumption [7].

Globally, British Columbia (BC), Canada, New South Wales (NSW), Australia, and Scotland are among the few settings with established surveillance systems that enable monitoring people with $\mathrm{HCV}$ infection, by linkage between $\mathrm{HCV}$ diagnosis databases and hospitalisation records. The aim of this study was to evaluate the contribution of AUD to population level decompensated cirrhosis (DC) diagnosis among people with an HCV notification. 


\section{Materials and methods}

Data sources and record linkages

BC, Canada

The British Columbia Hepatitis Testers Cohort (BC-HTC) holds records of all individuals tested for $\mathrm{HCV}, \mathrm{HBV}$, and $\mathrm{HIV}$ at the $\mathrm{BC}$ Centre for Disease Control Public Health Laboratory, since 1992, or reported as a confirmed case of HCV, HBV, and HIV/AIDS, since 1990.[8] At the end of each episode of admission, all BC hospitals submit their hospitalisation records (including demographic, administrative, and diagnosis information) directly to the Canadian Institute of Health Information for inclusion in the Discharge Abstracts Database, since 1985 [9]. All hospitalisation records are coded using the $10^{\text {th }}$ revision of the Classification of Diseases and Related Health Problems (ICD-10), since 2001. Information on all deaths registered in BC are held by the BC Vital Statistics Agency, since 1985 [10]. Using personal health numbers assigned to each individual in BC, BC-HTC records were deterministically linked to hospitalisation and mortality datasets. Record linkages were carried out by the BC Centre for Disease Control and BC Ministry of Health $[8]$.

\section{NSW, Australia}

The NSW Notifiable Conditions Information Management System (NCIMS) holds records of all individual with positive $\mathrm{HCV}$ and $\mathrm{HBV}$ serology tests, notified of diagnoses via mandatory notification procedures, since 1991 [11]. National HIV Registry holds records of all notifications of HIV infection, since 1985 [12]. NSW Admitted Patient Data Collection covers all inpatient admissions from all hospitals in NSW, since 2001 [11]. Each hospitalisation record includes demographic, administrative and diagnosis information coded at discharge according to ICD-10 [11]. Information on all deaths registered in NSW are held 
by the Registry of Births, Deaths and Marriages, since 1993 [11]. Using demographic details (including full name, gender, date of birth, and address), probabilistic linkages of records between the NCIMS, hospitalisation, and mortality datasets were undertaken by the NSW Centre for Health Record Linkage [11].

\section{Scotland}

Health Protection Scotland holds records of all individuals with positive HCV and HBV serology tests since 1991 and 1998, respectively [13]. Notifications of HIV diagnosis have been recorded since 1981 [13]. Hospital admission data are available from the Scottish Morbidity Records, comprising all Scottish hospitalisations since 1981, including demographic, administrative and diagnosis information coded at the end of each episode of admission (according to ICD-10 since 1996) [13]. Information on all deaths registered in Scotland are held by the National Records of Scotland, since 1980 [14]. Using unique identifiers and demographic details (including forename initial, surname Soundex, gender, date of birth, and postcode district of residence), a combination of deterministic and probabilistic linkages of records between the $\mathrm{HCV}$ notifications, hospitalisation, and mortality datasets were undertaken by Information Services Division Scotland [13].

\section{Study period}

In $\mathrm{BC}, \mathrm{NSW}$, and Scotland, HCV notifications were extracted for the study period between 1 January 1995 and 31 December 2011, 2012, and 2013, respectively, and; linked hospitalisation records were extracted for the study period between 1 January 2001 and 31 December 2012, 2013, and 2014, respectively. In each setting, linked mortality records were extracted for the study period between 1 January 1995 and the latest date of available hospitalisation data. 


\section{Study outcome}

The primary outcome of interest was first-time hospitalisation due to DC. A hospital discharge diagnosis code (ICD-10) was used to infer diagnosis of DC; coded in either the principal or secondary diagnosis fields of a linked inpatient hospital record. The set of relevant codes included: ascites (R18), bleeding oesophageal varices (I85.0 and I98.3), chronic hepatic failure (including hepatic encephalopathy) (K72.1 and K72.9), alcoholic hepatic failure (K70.4), and hepatorenal syndrome (K76.7). Hereafter, a first-time DC hospital admission is referred to as DC diagnosis.

\section{Exclusion criteria}

Exclusion criteria were applied as follows: records where date of birth was missing, and records where the date of $\mathrm{HCV}$ notification occurred more than three months after date of death (post-mortem notification), or prior to January 1, 1995.

\section{Statistical analysis}

Among people with an HCV notification in each setting, trends in DC diagnosis numbers and age at DC diagnosis were evaluated. Age-standardised DC incidence rates [per 100 personyears (PY)], and corresponding 95\% CIs were calculated assuming a Poisson distribution, overall and stratified by AUD. The European Standard Population 2013 was used for standardisation. The strength of association between risk factors and DC diagnosis was assessed using unadjusted and adjusted Cox proportional hazard regression analyses; covariates included birth cohort, gender, year of HCV notification, HBV and HIV coinfection, and AUD. The population attributable fractions (PAFs) and 95\% CIs of DC diagnosis associated with AUD were computed based on the adjusted regression analyses, overall and stratified by birth in or after 1965 [15]. 
AUD is a standard term used to define continued drinking despite adverse mental and physical consequences [16]. Liver-related consequences of alcohol use are not included in the definition of AUD [16]. A hospital discharge diagnosis code (ICD-10) at any point prior to DC diagnosis was used to infer the presence of AUD; coded in either the principal or a secondary diagnosis field of a linked inpatient hospital record. The set of relevant codes included: alcohol-induced Pseudo-Cushing's syndrome (E24.4), mental and behavioural disorders due to use of alcohol (F10), degeneration of nervous system due to alcohol (G31.2), alcoholic polyneuropathy (G62.1), alcoholic cardiomyopathy (I42.6), alcoholic myopathy (G72.1), alcohol rehabilitation (Z50.2), and alcohol abuse counselling and surveillance (Z71.4). Hereafter, having a history of at least one AUD-related hospital admission is referred to as AUD. AUD was not included as a time-dependent variable in Cox proportional regression analyses, given that it is considered to be an indicator of a long-standing condition. To calculate age-standardised DC incidence rates, assess factors associated with DC diagnosis, and evaluate PAFs, person-time at risk was defined to start six months post the date of HCV notification, and to end at whichever occurred first; death, or end of follow-up, assigned by year. Statistical analyses were carried out in STATA versions 12 and 13 or SAS version 9.4.

\section{Role of the funding source}

The funding sources did not have any role in study design, data collection and analysis, interpretation of data, writing of the report, and the decision to submit the paper for publication. The corresponding author had full access to all data in the study and had final responsibility for the decision to submit for publication. 


\section{Results}

\section{Study participants}

There were 58,487 people with an HCV notification in BC, 84,529 in NSW, and 31,924 in Scotland (total $\mathrm{n}=174,940)$ during the study period. The BC population was older $(70 \%$ born before 1965), compared to NSW (47\%) and Scotland (28\%). The proportion of people with AUD was highest in Scotland (27\%), followed by BC (19\%), and NSW (18\%). There were $2,689(4.6 \%)$ people with a DC diagnosis in BC, 3,169 (3.7\%) in NSW and 1,375 (4.3\%) in Scotland. Across the three settings, those with a DC diagnosis were more likely to be born during 1945-1964 and male. The proportion of people with a DC diagnosis and AUD was highest in Scotland (50\%), followed by NSW (32\%), and BC (28\%) (Table 1).

Age at DC diagnosis

The age distribution at DC diagnosis varied across settings, with median age of 54 years [interquartile range (IQR) 48, 60] in BC, 50 years (IQR 45, 56) in NSW, and 46 years (IQR $39,53)$ in Scotland. However, in all settings, compared to those without AUD, people with AUD had a younger age distribution, with median age of 52 vs. 56 years $(P<0.001)$ at DC diagnosis in $\mathrm{BC} ; 48$ vs. 52 years $(P<0.001)$ in NSW and; 43 vs. 49 years $(P<0.001)$ in Scotland (Figure 1).

\section{DC diagnosis numbers and rates}

In BC, NSW, and Scotland, DC diagnosis numbers increased from 196, 192, and 68 in 2004 to 295 in $2011(P<0.001), 351$ in $2012(P<0.001)$, and 157 in $2013(P<0.001)$, respectively. Among people with AUD in the three settings, DC diagnosis numbers increased from 52, 57, and 36 in 2004 to 88 in $2011(P=0.025), 145$ in $2012(P=0.001)$, and 72 in $2013(P=0.003)$, respectively. Since 2004, age-standardised DC incidence rates have remained relatively stable 
in all settings, although consistently higher among those with AUD in NSW and Scotland (Figure 2).

\section{Factors associated with DC diagnosis}

In unadjusted analyses, factors associated with DC diagnosis were consistent across all three settings. DC diagnosis was associated with older age, male gender, more recent HCV notification years $(\geq 2007)$, HIV and HBV/HIV co-infection, and AUD. In unadjusted analyses, HBV co-infection was associated with DC diagnosis in BC and NSW (Table 2). Similar demographic and clinical factors were associated with DC diagnosis in adjusted analyses (Table 3). The association between DC diagnosis and more recent HCV notification years was driven by older age (Supplementary Table 1). In adjusted analyses across the three settings, AUD independently predicted DC diagnosis [adjusted hazard ratio (aHR) BC 1.92, 95\% CI 1.76, 2.10; NSW 3.68, 95\% CI 3.38, 4.00; and Scotland 3.88, 95\% CI 3.42, 4.40] (Table 3).

Contribution of alcohol dependency to DC diagnosis

Overall, in BC, NSW, and Scotland, AUD was associated with a PAF of $13 \%$ (95\% CI 11\%, $15 \%), 25 \%(95 \%$ CI $23 \%, 27 \%)$, and $40 \%(95 \%$ CI $36 \%, 44 \%)$ of DC diagnoses, respectively. Among people born in or after 1965 in BC, NSW, and Scotland AUD was associated with a PAF of $21 \%(95 \%$ CI $16 \%, 25 \%), 36 \%(95 \%$ CI $32 \%, 40 \%)$, and $48 \%$ (95\% CI 43\%, 53\%) of DC diagnoses, respectively (Table 4). 


\section{Discussion}

This study outlines the rising absolute burden of HCV-related liver disease in BC, Canada; NSW, Australia; and Scotland. The increasing DC diagnosis numbers highlight the limited impact of interferon-containing HCV treatment programs across the three settings, as well as the combined effects of ageing and co-morbidities for liver disease progression. The impact of alcohol on liver disease progression among people with chronic $\mathrm{HCV}$ is evident through the younger age distribution of those with DC and AUD, and the higher attributable fractions for AUD among younger people with DC diagnosis. The overall contribution of AUD to DC diagnosis was highest in Scotland, the setting with the youngest age distribution, and lowest in $\mathrm{BC}$, consistent with the older age demographic in this setting. Elimination of HCV infection as a public health threat by 2030 is defined by a $65 \%$ reduction in liver-related mortality and an $80 \%$ reduction in incidence compared with the 2015 baseline [2]. To reach the $65 \%$ mortality reduction component of the elimination target, concentrated policy action is required to enhance HCV treatment uptake, and where needed, improve AUD management at the national level. The availability of data on mandatory HCV notifications and the capacity for regular linkage to other administrative databases provides the opportunity for ongoing evaluation and comparison of $\mathrm{HCV}$ public health strategies in the DAA era between BC, NSW, and Scotland.

Across the three settings, suboptimal efficacy and uptake of interferon-based therapies were among the major gaps in the HCV care cascade during the 2000s-early 2010s [1]. Over the coming decade, enhanced uptake of highly effective interferon-free DAA therapies is expected to significantly reduce the burden of HCV-related liver disease [17]; however, the impact of different strategies needs to be closely monitored. Australia has adopted a broad DAA access strategy without liver disease stage or drug and alcohol restrictions since March 
2016. Disease staging has been required for access to new therapies in BC and Scotland in 2016; however, eligibility criteria are opening to all disease stages as numbers of people with advanced liver disease diminish. Nonetheless, HCV-related liver disease is a multifactorial problem, to which antiviral therapy is only a partial solution [18]. The anticipated preventative impact of improved therapies has to be evaluated through linkage to individuallevel treatment data, across the three settings. Such comparison should be possible in the near future, following procedures to enable access to government-subsidised treatment datasets.

The major contribution of AUD to DC diagnosis has important policy implications in all settings. Strong evidence exists for the effectiveness of several strategies to reduce harmful consumption at the population level, including those targeting the pricing, availability and marketing of alcohol [19]. Canada is among a handful of countries that has implemented and evaluated the impact of different forms of minimum alcohol pricing [20]. Increasing minimum prices in Canada have been shown to be associated with significant reductions in harmful patterns of use, and alcohol-related hospitalisation and mortality [21-23]. In most countries however, governments fail to follow the evidence on alcohol management policies, given lack of popular support and difficulties of implementation [19]. Intricate patterns of consumption and alcohol-related harm further complicate the application and evaluation of management policies. In Scotland, alcohol-related morbidity and mortality have declined in recent years; however, these declines are not consistently distributed among the population, with the greatest burden recorded among those living in the most deprived areas [24]. In Australia, overall levels of drinking have declined during the last decade; however, diverging drinking patterns indicate these declines are mostly concentrated among lighter drinkers, while the top ten percent of heavier drinkers are responsible for an increasing proportion of total consumption [25]. Despite these differences, in developing country-specific 
management policies, tackling harmful consumption should remain integral to improving population level health outcomes and reducing social inequalities. After a prolonged battle with the well-organised and well-funded alcohol industry groups, Scotland has successfully passed legislation to introduce minimum unit pricing for alcohol. Ten years after implementation of this policy, the Scottish government estimates there would be at least 300 fewer alcohol-related deaths and 6,500 fewer hospital admissions each year [24]. In Australia, debates about minimising alcohol-related harm have increased in recent years [26, 27], instigating the Commonwealth government to explore the concept of a public interest case for minimum pricing of alcohol [28]. However, major alcohol policy reforms are yet to be implemented by the federal and state governments.

Across the three settings, older age was the strongest predictor of DC diagnosis. The causal relationship between ageing and increased risk of HCV-related complications is likely explained by a combination of longer duration of infection and ageing-related impaired repair mechanisms [29]. People born prior to 1945 were particularly at higher risk of developing DC. Interestingly, a recent study has demonstrated major acceleration in the development of HCV-related cirrhosis after the age of 50, irrespective of duration of infection [29]. Other predictors of DC diagnosis included AUD, male gender, and HBV/HCV/HIV co-infection. These characteristics are among well-defined risk factors for faster liver disease progression [30]. Defining an individual's risk of liver disease progression is often difficult, due to the non-linear nature of disease progression, and fluctuating lifestyle co-factors [31]. However, heavy alcohol use (>50 g/d) has been shown to be associated with end-stage liver disease diagnosis and liver-related mortality, independent of chronic $\mathrm{HCV}$ infection or viral eradication by therapy [32]. Given its significant impact, where needed, effects of AUD should be mitigated in order to lower the risk of liver disease progression among people with 
$\mathrm{HCV}$ infection. Integration of alcohol dependency treatment in medical settings could optimise management of AUD and facilitate provision of treatment by multidisciplinary teams, including psychosocial interventions, pharmacological therapy, and medical management of AUD and liver disease [33].

This study has several limitations. First, DC diagnosis was defined by a limited number of conditions that appear to be strong indicators of the decompensated stage of cirrhosis; however, this definition has not been validated against the clinical diagnoses of DC and requires further validation studies. Second, using administrative data for defining AUD has clear limitations. In sensitivity analysis, across the three settings, $72-94 \%$ of people who had a first-time hospitalisation due to alcoholic liver failure also had a non-liver AUD-related admission; the majority of which (71-85\%) were recorded before the first-time alcoholic liver failure hospitalisation. Further, a previous population-based study in Scotland has shown moderate and higher levels of self-reported alcohol consumption and binge drinking are major risk factors for subsequent alcohol-related hospitalisation (based on ICD codes) [34]. Finally, a validation study of administrative data (based on ICD codes) vs. chart notations has demonstrated $68 \%$ sensitivity and $97 \%$ specificity for the administrative data-based diagnosis of alcohol problem drinking [35]. Positive predictive value was $87 \%$, and negative predictive value was $91 \%$. Taken together, these findings suggest the current study has potential underascertainment of AUD, with potential overestimation of the impact on DC risk if more severe forms of AUD were more likely to be classified. Third, to improve the accuracy of trends in DC diagnosis numbers and age-standardised rates across the settings, these data were displayed and analysed since 2004. In Scotland, the pre-2004 DC trends were overestimated, given low HCV diagnosis rates during the early-mid 2000s (62\% undiagnosed in 2006) [36]. Fourth, in the analysis of the association between DC diagnosis and more recent HCV 
notification years, this study could not evaluate the potential impact of HCV diagnosis among people with more advanced liver disease in recent years. Previous findings from BC and NSW suggested the number of individuals with a late HCV notification has been declining in the 2000s (defined by an HCV notification before, at the time, or within two years before DC diagnosis); however, in the late 2000 s, approximately $20 \%$ of people with HCV-related DC diagnosis still had a late $\mathrm{HCV}$ notification [37, 38]. Fifth, other factors might have contributed to lower age-standardised $\mathrm{DC}$ rates in $\mathrm{BC}$, including residual confounding (attributed by unmeasured co-morbidities), and the older age distribution in this setting compared to the standard population. Sixth, HCV diagnosis for surveillance reporting is generally based on anti-HCV antibody detection and does not require HCV RNA confirmation. Thus, an estimated $25 \%$ of $\mathrm{HCV}$ notifications would have undergone spontaneous HCV clearance. Finally, the impact of antiviral therapy on DC diagnosis has not been evaluated in this study. Nevertheless, a minority of people would have received HCV treatment during the study period. These limitations should not have a major impact on the study findings, given the surveillance definition and systems in the three settings have been stable throughout the study period.

In conclusion, this international comparison of population level data provides evidence for the rising absolute burden of HCV-related advanced liver disease in BC, NSW, and Scotland, highlighting the combined impact of suboptimal HCV treatment efficacy and uptake, aging, and significant contribution of AUD to DC diagnosis. These findings suggest, where needed, action against AUD should form a strong component of $\mathrm{HCV}$ public health strategies. Continued heavy alcohol intake is likely to impact potential benefits of DAA-based cure on individual-level liver disease progression and population-level liver disease burden. Use of administrative databases for surveillance, particularly with the addition of individual-level 
antiviral treatment data will be a valuable tool for ongoing evaluation and comparison of the impact of DAA-based therapies on the individual-level liver disease progression and population-level burden of $\mathrm{HCV}$, given differences in the epidemiology of $\mathrm{HCV}$ and $\mathrm{HCV}$ public health strategies across the three settings. 


\section{References}

[1] Collaborators TPOH. Global prevalence and genotype distribution of hepatitis C virus infection in 2015: a modelling study. The Lancet Gastroenterology \& Hepatology 2017;2:161-167.

[2] World Health Organization. Global health sector strategy on viral hepatitis 20162021. Towards ending viral hepatitis. [cited July 2017)]; Available from: www.who.int/hepatitis/strategy2016-2021/ghss-hep/en/

[3] Younossi Z, Zheng L, Stepanova M, Venkatesan C, Mir H. Moderate, excessive or heavy alcohol consumption: each is significantly associated with increased mortality in patients with chronic hepatitis C. Alimentary Pharmacology and Therapeutics 2013;37:703709.

[4] Aspinall EJ, Hutchinson SJ, Janjua NZ, Grebely J, Yu A, Alavi M, et al. Trends in mortality after diagnosis of hepatitis $\mathrm{C}$ virus infection: an international comparison and implications for monitoring the population impact of treatment. Journal of Hepatology 2015;62:269-277.

[5] Grebely J, Haire B, Taylor LE, Macneill P, Litwin AH, Swan T, et al. Excluding people who use drugs or alcohol from access to hepatitis $\mathrm{C}$ treatments-Is this fair, given the available data? Journal of Hepatology 2015;63:779-782.

[6] Lazarus J, Safreed-Harmon K, Stumo S, Jauffret-Roustide M, Maticic M, Reic T, et al. Restrictions on access to direct-acting antivirals for people who inject drugs: The European Hep-CORE study and the role of patient groups in monitoring national HCV responses. International Journal of Drug Policy 2017 [In Press].

[7] Rehm J, Samokhvalov AV, Shield KD. Global burden of alcoholic liver diseases. Journal of Hepatology 2013;59:160-168.

[8] Janjua NZ, Kuo M, Chong M, Yu A, Alvarez M, Cook D, et al. Assessing hepatitis C burden and treatment effectiveness through the British Columbia Hepatitis Testers Cohort (BC-HTC): design and characteristics of linked and unlinked participants. PloS one 2016;11:e0150176.

[9] British Columbia Ministry of Health [creator]. Discharge Abstract Database (Hospital Separations). Victoria, BC: British Columbia Ministry of Health [publisher]; 2013. [cited June 2017)]; Available from: http://www2.gov.bc.ca/gov/content/health

[10] British Columbia Vital Statistics Agency [creator]. Vital Statistics Deaths. Victoria, BC: British Columbia Vital Statistics Agency [publisher]. Data Extract. British Columbia Vital Statistics Agency (2014). [cited June 2017)]; Available from: http://www2.gov.bc.ca/gov/content/life-events/death-and-bereavement

[11] The Centre for Health Record Linkage (CHeReL). [cited June 2017)]; Available from: http://www.cherel.org.au/

[12] McDonald AM, Crofts N, Blumer CE, Gertig DM, Patten JJ, Roberts M, et al. The pattern of diagnosed HIV infection in Australia, 1984-1992. Aids 1994;8:513-519.

[13] National Services Scotland. Information Services Division. [cited June 2017)]; Available from: www.isdscotland.org/index.asp

[14] National Records of Scotland. Births, Death and Marriage Records. [cited June 2017)]; Available from: www.nrscotland.gov.uk/research/guides/birth-death-and-marriagerecords

[15] Newson RB. Attributable and unattributable risks and fractions and other scenario comparisons. Stata Journal 2013;13:672-698.

[16] Friedmann PD. Alcohol use in adults. New England Journal of Medicine 2013;368:365-373. 
[17] Dore G, Ward J, Thursz M. Hepatitis C disease burden and strategies to manage the burden (Guest Editors Mark Thursz, Gregory Dore and John Ward). Journal of Viral Hepatitis 2014;21:1-4.

[18] Innes H, Hutchinson SJ, Obel N, Christensen PB, Aspinall EJ, Goldberg D, et al. Liver mortality attributable to chronic hepatitis $\mathrm{C}$ virus infection in Denmark and ScotlandUsing spontaneous resolvers as the benchmark comparator. Hepatology 2016;63:1506-1516.

[19] Gilmore W, Chikritzhs T, Stockwell T, Jernigan D, Naimi T, Gilmore I. Alcohol: taking a population perspective. Nature Reviews Gastroenterology \& Hepatology 2016;13:426-434.

[20] Thompson K, Stockwell T, Wettlaufer A, Giesbrecht N, Thomas G. Minimum alcohol pricing policies in practice: a critical examination of implementation in Canada. Journal of Public Health Policy 2016 38:39-57.

[21] Stockwell T, Zhao J, Giesbrecht N, Macdonald S, Thomas G, Wettlaufer A. The raising of minimum alcohol prices in Saskatchewan, Canada: impacts on consumption and implications for public health. American Journal of Public Health 2012;102:e103-e110.

[22] Stockwell T, Auld MC, Zhao J, Martin G. Does minimum pricing reduce alcohol consumption? The experience of a Canadian province. Addiction 2012;107:912-920.

[23] Zhao J, Stockwell T, Martin G, Macdonald S, Vallance K, Treno A, et al. The relationship between minimum alcohol prices, outlet densities and alcohol-attributable deaths in British Columbia, 2002-09. Addiction 2013;108:1059-1069.

[24] The Scottish Government. The Scottish Health Survey 2014: Volume 1: Main Report, Page 318753. [cited March 2017)]; Available from: http://www.gov.scot/Publications/2015/09/6648/318753

[25] Livingston, M. Understanding recent trends in Australian alcohol consumption. Canberra: Foundation for Alcohol Research and Education. 2015 [cited March 2017)]; Available from: $\quad$ http://www.smh.com.au/cqstatic/gm9lbv/Livingston\%20\%20Understanding-recent-trends-in-Australian-alcohol-consumpti....pdf

[26] Chalmers J, Carragher N, Davoren S, O'Brien P. Real or perceived impediments to minimum pricing of alcohol in Australia: public opinion, the industry and the law. International Journal of Drug Policy 2013;24:517-523.

[27] Buykx P, Gilligan C, Ward B, Kippen R, Chapman K. Public support for alcohol policies associated with knowledge of cancer risk. International Journal of Drug Policy 2015;26:371-379.

[28] Australian National Preventive Health Agency. Exploring the public interest case for a minimum (floor) price for alcohol. 2013 [cited March 2017)]; Available from: http://health.gov.au/internet/anpha/publishing.nsf/Content/minimum-price-final-report

[29] El-Serag H, Kramer J, Duan Z, Kanwal F. Epidemiology and outcomes of hepatitis C infection in elderly US Veterans. Journal of Viral Hepatitis 2016;23:687-696.

[30] Westbrook RH, Dusheiko G. Natural history of hepatitis C. Journal of Hepatology 2014;61:S58-S68.

[31] Thein HH, Yi Q, Dore GJ, Krahn MD. Estimation of stage-specific fibrosis progression rates in chronic hepatitis $\mathrm{C}$ virus infection: A meta-analysis and meta-regression. Hepatology 2008;48:418-431.

[32] McMahon BJ, Bruden D, Bruce MG, Livingston S, Christensen C, Homan C, et al. Adverse outcomes in Alaska natives who recovered from or have chronic hepatitis $\mathrm{C}$ infection. Gastroenterology 2010;138:922-931. e921.

[33] Addolorato G, Mirijello A, Barrio P, Gual A. Treatment of Alcohol Use Disorders in patients with Alcoholic Liver Disease. Journal of Hepatology 2016 65:618-630. 
[34] McDonald SA, Hutchinson SJ, Bird SM, Graham L, Robertson C, Mills PR, et al. Association of self-reported alcohol use and hospitalization for an alcohol-related cause in Scotland: a record-linkage study of 23183 individuals. Addiction 2009;104:593-602.

[35] Kim HM, Smith EG, Stano CM, Ganoczy D, Zivin K, Walters H, et al. Validation of key behaviourally based mental health diagnoses in administrative data: suicide attempt, alcohol abuse, illicit drug abuse and tobacco use. BMC health services research 2012;12:18.

[36] McLeod A, Weir A, Aitken C, Gunson R, Templeton K, Molyneaux P, et al. Rise in testing and diagnosis associated with Scotland's Action Plan on Hepatitis $\mathrm{C}$ and introduction of dried blood spot testing. Journal of Epidemiology and Community Health 2014;68:11821188.

[37] Alavi M, Law MG, Grebely J, Amin J, Hajarizadeh B, George J, et al. Time to decompensated cirrhosis and hepatocellular carcinoma after an HBV or HCV notification: A population-based study. Journal of Hepatology 2016;65:879-887.

[38] Samji H, Yu A, Kuo M, Alavi M, Woods R, Alvarez M, et al. Late hepatitis B and C diagnosis in relation to disease decompensation and hepatocellular carcinoma development. Journal of Hepatology 2017 [In Press]. 
Table 1. Demographic characteristics among people with an HCV notification in BC,

NSW, and Scotland, since 1995

\begin{tabular}{|c|c|c|c|c|c|c|c|c|c|c|c|c|}
\hline \multirow[b]{3}{*}{ Characteristics, n \% } & \multicolumn{4}{|c|}{$\begin{array}{c}\text { BC, Canada } \\
\text { HCV notifications } \\
\text { 1995-2011 }\end{array}$} & \multicolumn{3}{|c|}{$\begin{array}{c}\text { NSW, Australia } \\
\text { HCV notifications } \\
\text { 1995-2012 }\end{array}$} & \multicolumn{5}{|c|}{$\begin{array}{c}\text { Scotland } \\
\text { HCV notifications } \\
1995-2013 \\
\end{array}$} \\
\hline & All & & $\mathbf{D C}^{\alpha}$ & & All & & $\mathrm{DC}^{\alpha}$ & & All & & $\mathbf{D C}^{\alpha}$ & \\
\hline & $\mathrm{n}=58,487$ & $\%$ & $\mathrm{n}=2,689$ & $\%$ & $\mathrm{n}=84,529$ & $\%$ & $n=3,169$ & $\%$ & $\mathrm{n}=31,924$ & $\%$ & $\mathrm{n}=1,375$ & $\%$ \\
\hline \multicolumn{13}{|l|}{ Birth cohort } \\
\hline$\geq 1965$ & 17,397 & 30 & 210 & 8 & 45,298 & 54 & 611 & 19 & 22,889 & 72 & 602 & 44 \\
\hline 1945-1964 & 35,616 & 61 & 1,981 & 74 & 34,518 & 41 & 2,171 & 69 & 8,051 & 25 & 681 & 50 \\
\hline$\leq 1944$ & 5,474 & 9 & 498 & 19 & 4,713 & 6 & 387 & 12 & 984 & 3 & 92 & 7 \\
\hline Male $^{\beta}$ & 38,212 & 65 & 1,860 & 69 & 53,146 & 63 & 2,319 & 73 & 21,369 & 65 & 1,002 & 73 \\
\hline \multicolumn{13}{|l|}{$\begin{array}{l}\text { Year of HCV } \\
\text { notification }\end{array}$} \\
\hline $1995-2000$ & 28,517 & 49 & 1,449 & 54 & 39,043 & 46 & 1,461 & 46 & 9,141 & 29 & 377 & 27 \\
\hline 2001-2006 & 18,339 & 31 & 920 & 34 & 26,754 & 32 & 1,088 & 34 & 9,645 & 30 & 545 & 40 \\
\hline 2007-2011/12/13 & 11,631 & 20 & 320 & 12 & 18,732 & 22 & 620 & 20 & 13,138 & 41 & 453 & 33 \\
\hline \multicolumn{13}{|l|}{ Co-infection status } \\
\hline None & 51,381 & 88 & 2,241 & 83 & 80,112 & 95 & 2,934 & 93 & 30,653 & 96 & 1,289 & 94 \\
\hline HBV & 3,255 & 6 & 229 & 9 & 3,467 & 4 & 184 & 6 & 587 & 2 & 41 & 3 \\
\hline HIV & 2,910 & 5 & 150 & 6 & 888 & 1 & 45 & 1 & 645 & 2 & 40 & 3 \\
\hline HBV/HIV & 941 & 2 & 69 & 3 & 62 & $<1$ & 6 & $<1$ & 39 & $<1$ & 5 & $<1$ \\
\hline $\begin{array}{l}\text { Alcohol-use } \\
\text { disorder }^{\gamma}\end{array}$ & 11,078 & 19 & 742 & 28 & 14,797 & 18 & 1,023 & 32 & 8,757 & 27 & 693 & 50 \\
\hline
\end{tabular}

${ }^{\alpha}$ DC diagnosis during 2001-2012 in BC, 2001-2013 in NSW, and 2001-2014 in Scotland, ${ }^{\beta}$ among people with available information, ${ }^{\gamma}$ among people with a DC diagnosis, Alcohol-use disorder was included if occurred prior to DC diagnosis 
Table 2. Unadjusted analysis of factors associated with DC diagnosis among people with an HCV notification in BC, NSW, and

\section{Scotland, since 1995}

\begin{tabular}{|c|c|c|c|c|c|c|c|c|c|c|c|c|c|c|c|}
\hline \multirow[b]{2}{*}{ Characteristics, n \% } & \multicolumn{5}{|c|}{$\begin{array}{c}\text { BC, Canada } \\
\text { HCV notification, 1995-2011 } \\
\text { n=55,873 }\end{array}$} & \multicolumn{5}{|c|}{$\begin{array}{c}\text { NSW, Australia } \\
\text { HCV notification, 1995-2012 } \\
\text { n=82,526 } \\
\end{array}$} & \multicolumn{5}{|c|}{$\begin{array}{c}\text { Scotland } \\
\text { HCV notification, } 1995-2013 \\
n=30,746 \\
\end{array}$} \\
\hline & $\begin{array}{c}C^{\alpha} \\
n=2,443\end{array}$ & $\%$ & $\mathrm{HR}^{\beta}$ & $95 \% \mathrm{CI}$ & $P$ & $\begin{array}{c}D^{\prime} C^{\alpha} \\
n=2,559\end{array}$ & $\%$ & $\mathrm{HR}^{\beta}$ & $95 \% \mathrm{CI}$ & $\boldsymbol{P}$ & $\begin{array}{c}\mathbf{D C}^{\alpha} \\
\mathbf{n}=\mathbf{1 , 0 2 0}\end{array}$ & $\%$ & $\mathrm{HR}^{\beta}$ & $95 \%$ CI & $P$ \\
\hline \multicolumn{16}{|l|}{ Birth cohort } \\
\hline$\geq 1965$ & 198 & 1 & 1.00 & - & - & 479 & 1 & 1.00 & - & - & 466 & 2 & 1.00 & - & - \\
\hline 1945-1964 & 1,804 & 5 & 4.21 & $3.64,4.88$ & $<0.001$ & 1,750 & 5 & 4.59 & $6.81,9.01$ & $<0.001$ & 490 & 7 & 2.97 & $2.61,3.37$ & $<0.001$ \\
\hline$\leq 1944$ & 441 & 10 & 9.18 & $7.77,10.86$ & $<0.001$ & 330 & 8 & 7.83 & $4.15,5.08$ & $<0.001$ & 64 & 8 & 4.45 & $3.43,5.79$ & $<0.001$ \\
\hline \multicolumn{16}{|l|}{ Gender } \\
\hline Female & 749 & 4 & 1.00 & - & - & 690 & 4 & 1.00 & - & - & 287 & 3 & 1.00 & - & - \\
\hline Male & 1,694 & 5 & 1.24 & $1.14,1.35$ & $<0.001$ & 1,860 & 4 & 1.63 & $1.50,1.78$ & $<0.001$ & 732 & 4 & 1.28 & $1.11,1.46$ & $<0.001$ \\
\hline \multicolumn{16}{|l|}{$\begin{array}{l}\text { Year of HCV } \\
\text { notification }\end{array}$} \\
\hline 1995-2000 & 1,443 & 5 & 1.00 & - & - & 1,355 & 4 & 1.00 & - & - & 274 & 4 & 1.00 & - & - \\
\hline 2001-2006 & 792 & 4 & 1.14 & $1.04,1.25$ & 0.003 & 879 & 3 & 1.11 & $1.01,1.23$ & 0.032 & 397 & 4 & 1.28 & $1.09,1.51$ & 0.002 \\
\hline$\geq 2007$ & 208 & 2 & 1.31 & $1.12,1.53$ & 0.001 & 325 & 2 & 1.51 & $1.30,1.75$ & $<0.001$ & 249 & 2 & 1.42 & $1.16,1.75$ & 0.001 \\
\hline \multicolumn{16}{|l|}{ Co-infection status } \\
\hline None & 2016 & 4 & 1.00 & - & - & 2,387 & 3 & 1.00 & - & - & 958 & 3 & 1.00 & - & - \\
\hline HBV & 214 & 7 & 1.60 & $1.39,1.84$ & $<0.001$ & 132 & 4 & 1.35 & $1.13,1.61$ & 0.001 & 26 & 5 & 1.39 & $0.94,2.05$ & 0.100 \\
\hline HIV & 144 & 5 & 1.32 & $1.12,1.57$ & $<0.001$ & 36 & 4 & 1.66 & $1.20,2.31$ & 0.002 & 31 & 5 & 1.82 & $1.27,2.60$ & 0.001 \\
\hline HBV/HIV & 69 & 8 & 2.01 & $1.58,2.56$ & $<0.001$ & 4 & 7 & 3.57 & $1.34,9.53$ & 0.011 & 5 & 14 & 5.48 & $2.27,13.19$ & $<0.001$ \\
\hline \multicolumn{16}{|l|}{ Alcohol-use disorder ${ }^{\gamma}$} \\
\hline No & 1,775 & 4 & 1.00 & - & - & 1,672 & 2 & 1.00 & - & - & 464 & 2 & 1.00 & - & - \\
\hline Yes & 668 & 7 & 1.82 & $1.67,1.99$ & $<0.001$ & 887 & 6 & 2.96 & $2.73,3.21$ & $<0.001$ & 556 & 7 & 3.62 & $3.20,4.10$ & $<0.001$ \\
\hline
\end{tabular}


Table 3. Adjusted analysis of factors associated with DC diagnosis among people with an HCV notification in BC, NSW, and Scotland,

since 1995

\begin{tabular}{|c|c|c|c|c|c|c|c|c|c|c|c|c|c|c|c|}
\hline \multirow[b]{2}{*}{ Characteristics, n \% } & \multicolumn{5}{|c|}{$\begin{array}{c}\text { BC, Canada } \\
\text { HCV notification, 1995-2011 } \\
\text { n=55,873 }\end{array}$} & \multicolumn{5}{|c|}{$\begin{array}{c}\text { NSW, Australia } \\
\text { HCV notification, 1995-2012 } \\
\text { n=82,526 } \\
\end{array}$} & \multicolumn{5}{|c|}{$\begin{array}{c}\text { Scotland } \\
\text { HCV notification, 1995-2013 } \\
\text { n=30,746 }\end{array}$} \\
\hline & $\begin{array}{c}D^{\prime a} C^{\alpha} \\
n=2,443\end{array}$ & $\%$ & $\mathbf{a H R}^{\beta}$ & $95 \% \mathrm{CI}$ & $P$ & $\begin{array}{c}D^{\prime a} C^{\alpha} \\
n=2,559\end{array}$ & $\%$ & $\mathbf{a H R}^{\beta}$ & $95 \% \mathrm{CI}$ & $P$ & $\begin{array}{c}D^{D} C^{\alpha} \\
n=1,020\end{array}$ & $\%$ & $\mathbf{a H R}^{\beta}$ & $95 \% \mathrm{CI}$ & $P$ \\
\hline \multicolumn{16}{|l|}{ Birth cohort } \\
\hline$\geq 1965$ & 198 & 1 & 1.00 & - & - & 479 & 1 & 1.00 & - & - & 466 & 2 & 1.00 & - & - \\
\hline 1945-1964 & 1,804 & 5 & 4.55 & $3.93,5.28$ & $<0.001$ & 1,750 & 5 & 5.13 & $4.63,5.69$ & $<0.001$ & 490 & 7 & 3.07 & $2.70,3.49$ & $<0.001$ \\
\hline$\leq 1944$ & 441 & 10 & 11.13 & $9.40,13.19$ & $<0.001$ & 330 & 8 & 11.90 & $10.30,13.75$ & $<0.001$ & 64 & 8 & 7.09 & $5.43,9.25$ & $<0.001$ \\
\hline \multicolumn{16}{|l|}{ Gender } \\
\hline Female & 749 & 4 & 1.00 & - & - & 690 & 4 & 1.00 & - & - & 287 & 3 & 1.00 & - & - \\
\hline Male & 1,694 & 5 & 1.15 & $1.06,1.26$ & 0.001 & 1,860 & 4 & 1.43 & $1.31,1.56$ & $<0.001$ & 732 & 4 & 1.15 & $1.00,1.32$ & 0.048 \\
\hline \multicolumn{16}{|l|}{$\begin{array}{l}\text { Year of HCV } \\
\text { notification }\end{array}$} \\
\hline 1995-2000 & 1,443 & 5 & 1.00 & - & - & 1,355 & 4 & 1.00 & - & - & 274 & 4 & 1.00 & - & - \\
\hline 2001-2006 & 792 & 4 & 1.36 & $1.24,1.49$ & $<0.001$ & 879 & 3 & 1.25 & $1.13,1.37$ & $<0.001$ & 397 & 4 & 1.46 & $1.24,1.72$ & $<0.001$ \\
\hline$\geq \mathbf{2 0 0 7}$ & 208 & 2 & 1.81 & $1.55,2.12$ & $<0.001$ & 325 & 2 & 1.87 & $1.61,2.17$ & $<0.001$ & 249 & 2 & 1.81 & $1.48,2.23$ & $<0.001$ \\
\hline \multicolumn{16}{|l|}{ Co-infection status } \\
\hline None & 2016 & 4 & 1.00 & - & - & 2,387 & 3 & 1.00 & - & - & 958 & 3 & 1.00 & - & - \\
\hline HBV & 214 & 7 & 1.55 & $1.35,1.79$ & $<0.001$ & 132 & 4 & 1.40 & $1.17,1.67$ & $<0.001$ & 26 & 5 & 1.34 & $0.94,1.99$ & 0.138 \\
\hline HIV & 144 & 5 & 1.55 & $1.31,1.84$ & $<0.001$ & 36 & 4 & 1.61 & $1.16,2.24$ & 0.005 & 31 & 5 & 1.50 & $1.04,2.14$ & 0.028 \\
\hline HBV/HIV & 69 & 8 & 2.37 & $1.86,3.02$ & $<0.001$ & 4 & 7 & 2.66 & $1.00,7.10$ & 0.051 & 5 & 14 & 5.50 & $2.28,13.27$ & $<0.001$ \\
\hline \multicolumn{16}{|l|}{ Alcohol-use disorder ${ }^{\gamma}$} \\
\hline No & 1,775 & 4 & 1.00 & - & - & 1,672 & 2 & 1.00 & - & - & 464 & 2 & 1.00 & - & - \\
\hline Yes & 688 & 7 & 1.92 & $1.76,2.10$ & $<0.001$ & 887 & 6 & 3.68 & $3.38,4.00$ & $<0.001$ & 556 & 7 & 3.88 & $3.42,4.40$ & $<0.001$ \\
\hline
\end{tabular}


Table 4. Population attributable fraction of DC diagnosis associated with alcohol-use disorder among BC, NSW, and Scotland people

with an HCV notification, since 1995

\begin{tabular}{|c|c|c|c|c|c|c|c|c|c|c|c|c|}
\hline & \multicolumn{4}{|c|}{$\begin{array}{l}\text { BC HCV notification, 1995-2011 } \\
\qquad n=55,879 \\
\text { DC diagnosis 2001-2012, } n=2,443\end{array}$} & \multicolumn{4}{|c|}{$\begin{array}{l}\text { NSW HCV notification, 1995-2012 } \\
n=82,526 \\
\text { DC diagnosis 2001-2013, } n=2,559\end{array}$} & \multicolumn{4}{|c|}{$\begin{array}{l}\text { Scotland HCV notification, 1995-2013 } \\
\text { n=30,746 } \\
\text { DC diagnosis 2001-2014, } n=1,020\end{array}$} \\
\hline & $\begin{array}{l}\text { Alcohol-use } \\
\text { disorder, n }\end{array}$ & $\%$ & PAF $^{\alpha}$ & $95 \% \mathrm{CI}$ & $\begin{array}{l}\text { Alcohol-use } \\
\text { disorder, n }\end{array}$ & $\%$ & $\mathbf{P A F}^{\alpha}$ & $95 \%$ CI & $\begin{array}{l}\text { Alcohol-use } \\
\text { disorder, n }\end{array}$ & $\%$ & PAF $^{\alpha}$ & $95 \% \mathrm{CI}$ \\
\hline All & 688 & 7 & 13 & 11,15 & 887 & 6 & 25 & 23,27 & 556 & 7 & 40 & 36,44 \\
\hline Born $\geq 1965$ & 90 & 3 & 21 & 16,25 & 247 & 3 & 36 & 32,40 & 309 & 5 & 48 & 43,53 \\
\hline Born $<1965$ & 598 & 9 & 12 & 10,14 & 640 & 13 & 22 & 20,24 & 247 & 12 & 33 & 28,37 \\
\hline
\end{tabular}


Figure 1. Age at DC diagnosis among people with an HCV notification in BC, NSW, and Scotland, by alcohol-use disorder, since 1995
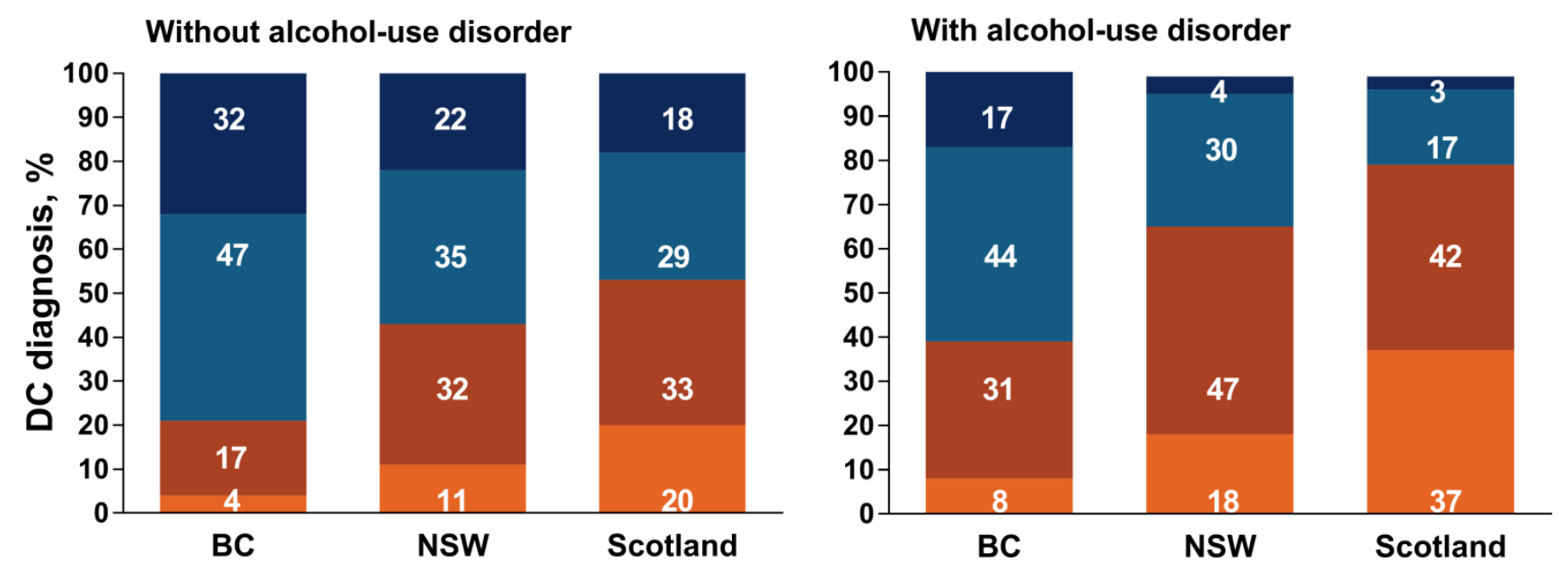

- 60+ years old at the time of DC diagnosis

- 50-59 years old at the time of DC diagnosis

- 40-49 years old at the time of DC diagnosis

$<40$ years old at the time of DC diagnosis 
Figure 2. Temporal trends in DC diagnosis numbers and age-standardised incidence rates among people with an HCV notification in BC, NSW, and Scotland, by alcohol-use disorder, since 1995
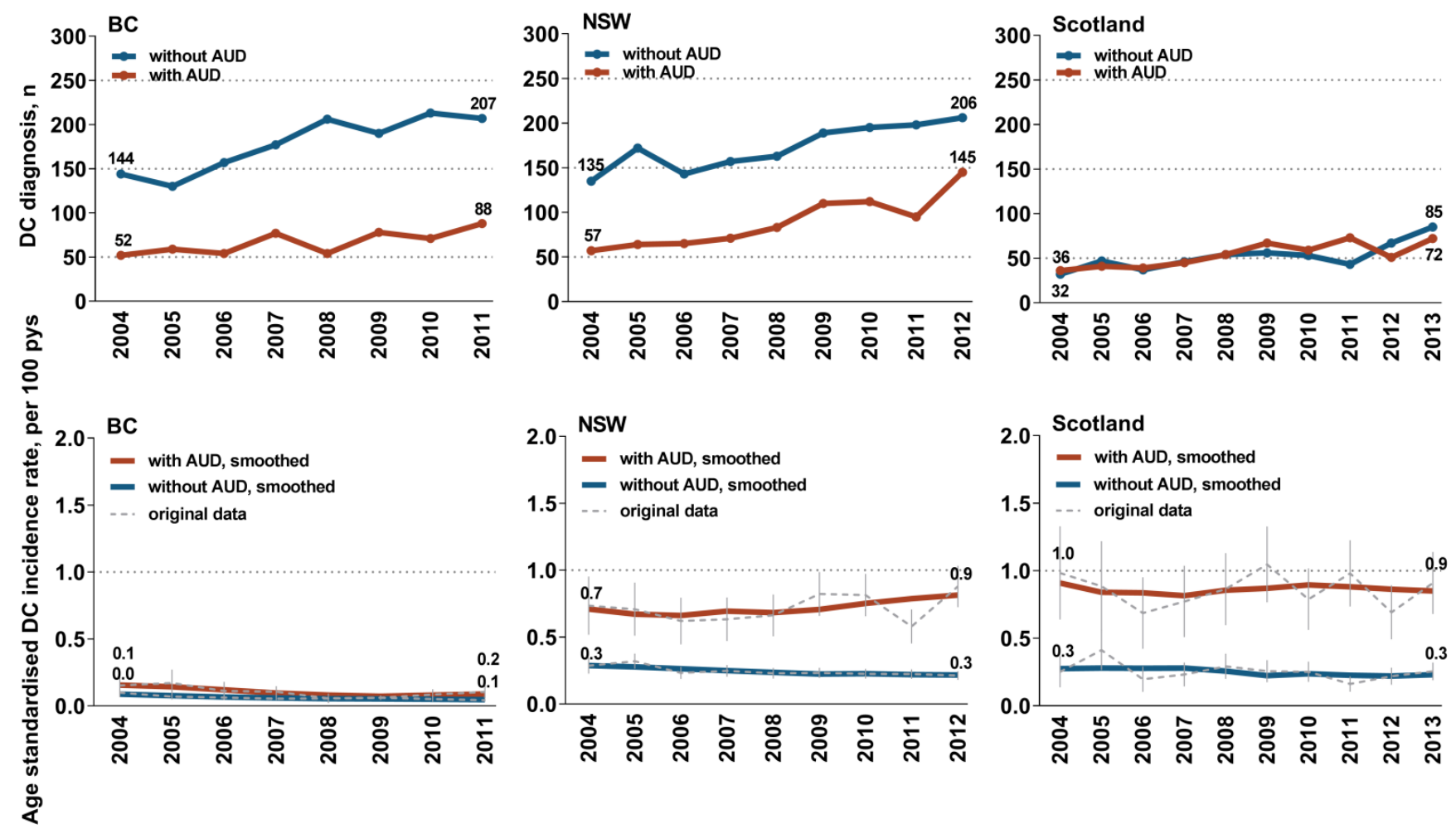